\title{
A SHORT PROOF OF A DECOMPOSITION THEOREM OF A VON NEUMANN ALGEBRA
}

\author{
M. AWAMI AND A. B. THAHEEM
}

\begin{abstract}
Let $M$ be a von Neumann algebra and $S$ and $T$ be commuting ${ }^{*}$-automorphisms on $M$ satisfying the equation: $S+S^{-1}=T+T^{-1}$. It is proved that $M$ can be decomposed by a central projection $p$ in $M$ such that $S=T$ on $M p$ and $S=T^{-1}$ on $M(1-p)$.
\end{abstract}

Recently the second author proved the following decomposition theorem.

THEOREM 1 [3, THEOREM 2.6]. Let $S$ and $T$ be commuting *-automorphisms of a von Neumann algebra $M$ satisfying the operator equation

$$
S+S^{-1}=T+T^{-1} .
$$

Then $M$ can be decomposed by a central projection $p$ in $M$ such that $S=T$ on $M p$ and $S=T^{-1}$ on $M(1-p)$.

Some applications of this result are discussed by Haagerup and Skau in the geometrical interpretation of the Tomita-Takesaki theory (cf. [1]). A noncommutative version of Theorem 1 (in the case of automorphism groups) has been studied in [4] with its proof depending on Arveson's theory of spectral subspaces (cf. [5]). The proof of Theorem 1 is rather long and relies on several technical lemmas. The purpose of this note is to give a short proof of this theorem. In fact, we prove a stronger form of this result (Theorem 2) and get the proof of Theorem 1 as an immediate corollary.

THEOREM 2. Let $S$ and $T$ be *-automorphisms of a von Neumann algebra $M$ satisfying the operator equation

$$
S+T^{-1} S^{-1} T=T+T^{-1} .
$$

Then $M$ can be decomposed by a central projection $p$ in $M$ such that $S=T$ on $M p$ and $S=T^{-1}$ on $M(1-p)$.

*Received by the editors December 14, 1982.

1980 Mathematics Subject Classification. Primary 46L10; Secondary 47C15.

Key words and phrases. Automorphisms, central projection.

(C)1984 American Mathematical Society $0002-9939 / 84 \$ 1.00+\$ .25$ per page 
Proof. Consider the ${ }^{*}$-automorphism $S^{-1} T$ on $M$. By [2, Theorem 2] there is a central projection $p$ in $M$ such that $M(1-p)$ is the smallest subalgebra generated by $R\left(S^{-1} T-1\right)$, the range of $\left(S^{-1} T-1\right)$, and $\left(S^{-1} T-1\right)(M p)=0$. Since

$$
\left(T^{-1}-S\right)\left(S^{-1} T-1\right)=T^{-1} S^{-1} T-T-T^{-1}+S=0 \quad(\text { by }(\mathrm{i})),
$$

therefore, $\left(T^{-1}-S\right)(M(1-p))=0$. This completes the proof of the theorem.

COROLlaRY. If $S$ and $T$ commute then we get the proof of Theorem 1.

\section{REFERENCES}

1. U. Haagerup and F. Skau, Geometric aspects of the Tomita-Takesaki theory. II, Math. Scand. 48 (1981), 241-252.

2. A. B. Thaheem, Decomposition of a von Neumann algebra relative to $a^{*}$-automorphism, Proc. Edinburgh Math. Soc. (2) 22 (1979), 9-10.

3. __ Decomposition of a von Neumann algebra, Rend. Sem. Mat. Univ. Padova 65 (1981), 1-7.

4. A. B. Thaheem, A. Van Daele and L. Vanheeswijck, $A$ result on two-one-parameter groups of automorphisms, Math. Scand. (to appear).

5. A. Van Daele, Arveson's theory of spectral subspaces, Nieuw Arch. Wisk. (3) 27 (1979), 215-237.

Department of Mathematics, Faculty of Science, Garyounis University, P. O. Box 9480 , BENGHAZI, LIBYA 